\title{
Knockdown of HMGN5 increases the chemosensitivity of human urothelial bladder cancer cells to cisplatin by targeting PI3K/Akt signaling
}

\author{
YU GAN, LEYE HE, KUN YAO, JING TAN, QING ZENG, YINGBO DAI, JIANYE LIU and YUXIN TANG
}

Department of Urology, The Third Xiangya Hospital of Central South University, Changsha, Hunan 410013, P.R. China

Received June 28, 2016; Accepted August 8, 2017

DOI: 10.3892/ol.2017.7045

\begin{abstract}
High-mobility group nucleosome-binding domain 5 (HMGN5) is the latest member of the HMGN family of proteins. Numerous studies have confirmed the carcinogenic role of HMGN5 in cancer, but its function in the regulation of chemosensitivity is largely unknown and controversial. A previous study by the authors of the present study demonstrated that HMGN5 contributes to the progression of urothelial bladder cancer (UBC) through regulating the expression of E-cadherin and vascular endothelial growth factor (VEGF)-C, which are associated with the sensitivity of tumor cells to cisplatin. Therefore, the present study aimed to elucidate the mechanisms underlying the regulation of HMGN5 and investigate the involvement of HMGN5 in cisplatin treatment. The results of the present study revealed that HMGN5 is able to positively regulate the expression of phosphorylated (p-)Akt in UBC cells. In addition, HMGN5 expression was negatively associated with the response of UBC cells to cisplatin. The findings indicated that HMGN5 may be a potential therapeutic target of cisplatin treatment, since cisplatin treatment reduced HMGN5 expression in a dose-dependent manner. It was also confirmed that the knockdown of HMGN5 decreased the viability, colony formation and invasion of 5637 cells but increased apoptosis under cisplatin treatment. The changes caused by HMGN5 knockdown in 5637 cells were able to be reversed by treatment with insulin-like growth factor-1 (IGF-1), which is a phosphoinositide 3-kinase (PI3K)/Akt signaling activator. Additionally, with the decreased expression of HMGN5, the expression of p-Akt, slug, E-cadherin and VEGF-C was subsequently inhibited. By contrast, the expression of cytochrome c, cleaved-caspase-3 and cleaved-poly ADP ribose polymerase was increased following HMGN5 knockdown. Consistently, these changes in protein
\end{abstract}

Correspondence to: Dr Kun Yao, Department of Urology, The Third Xiangya Hospital of Central South University, 138 Tongzipo Road, Changsha, Hunan 410013, P.R. China

E-mail: yaokun52@163.com

Key words: high-mobility group nucleosome-binding domain 5, urothelial bladder cancer, cisplatin, chemosensitivity, phosphoinositide 3-kinase, Akt expression were able to be reversed by IGF-1 treatment. In conclusion, findings from the in vitro experiments indicate that HMGN5 may a target of cisplatin treatment and that the inhibition of HMGN5 increases the chemosensitivity of UBC cells by inhibiting PI3K/Akt signaling.

\section{Introduction}

In Europe, urothelial bladder cancer (UBC) is the second most common malignancy in the genitourinary tract and the fifth most common type of cancer, with a high rate of morbidity and mortality $(1,2)$. The majority of initially diagnosed cases of $\mathrm{UBC}$ is non-muscle invasive and can be effectively treated by transurethral resection, combined with intravesical chemo- or immunotherapy (3). However, at the time of first diagnosis or at late visits, $\sim 30 \%$ of UBC cases exhibit an invasive growth pattern, being either muscle-invasive (pT2) or more advanced, such as locally advanced (pT3-4 and/or pN1-3 M0) or metastatic (M1) $(3,4)$. The overall prognosis of patients with UBC with this pattern remains poor. Cisplatin (cis-diaminodichloroplatinum, CDDP)-based chemotherapy is widely used in this group of patients and provides a survival advantage (5). However, the response rate to chemotherapy remains $\sim 50 \%$ (6). In consideration of function of CDDP in the currently employed chemotherapy regimens, the principal cause of poor response involves resistance to CDDP. However, thus far, the mechanisms underlying CDDP inhibition of UBC have not yet been fully elucidated, which represents a great challenge for physicians.

High-mobility group nucleosome-binding domain 5 (HMGN5), also termed NBP-45, GARP45 or NSBP1, is the latest member of the HMGN family of proteins, which was identified by King et al in 2001 (7,8). Since identification, the gene has been reported to primarily function in embryonic development, regulation of transcription and chromatin decompaction (8). In recent years, emerging studies have confirmed that HMGN5 is overexpressed in various human tumors and confers oncogenic effects in various cancer models (9). However, the effects of the gene on chemosensitivity to commonly used chemotherapy regimens in cancer cells remain largely unknown and controversial.

In a previous study by the present authors, it was revealed that knockdown of HMGN5 suppressed the viability and invasion of human UBC 5637 cells via regulating the expression of E-cadherin, a marker of epithelial-mesenchymal transition 
(EMT), and vascular endothelial growth factor (VEGF)-C, a marker of lymphangiogenesis $(10,11)$. It was reported that EMT and the transcription factor slug directly contribute to CDDP resistance $(12,13)$. In addition, Zhu et al (14) reported that inhibition of VEGF-C reversed resistance of UBC cells to CDDP. Therefore, the present study aimed to investigate the involvement of HMGN5 in the treatment of UBC using CDDP. However, more efforts are required to elucidate the role of HMGN5 in cancer progression of UBC.

The present study examined the function of HMGN5 on the sensitivity of UBC cells to CDDP in vitro and investigated the underlying mechanisms. Results of the present study demonstrated that the UBC cells expressing a low level of HMGN5 are more sensitive to CDDP, and CDDP suppresses the growth of UBC cells by inhibiting HMGN5. Furthermore, it was verified that HMGN5 depletion increases the sensitivity of UBC 5637 cells to CDDP via inhibiting PI3K/Akt signaling. These findings indicated that HMGN5 is a potential therapy target in UBC treatment.

\section{Materials and methods}

Cell culture, transfection and drug treatment. The human UBC 5637, UM-UC-3 and T24 cell lines were obtained from Yingrun Biotechnologies, Inc. (Changsha, China). The cells were maintained in RPMI-1640 (Thermo Fisher Scientific, Inc., Waltham, MA, USA) supplemented with $10 \%$ fetal calf serum (Thermo Fisher Scientific, Inc.) in a humidified 5\% $\mathrm{CO}_{2}, 37^{\circ} \mathrm{C}$ incubator.

HMGN5 short hairpin RNA (shRNA) sequences and construction of lentivirus were the same as described in a previous study by the present authors (10). Briefly, the most effective shRNA sequences targeting HMGN5 (5'-GTTGTT GAAGAAGACTACAAT-3') were synthesized and cloned into the pYr-Lvsh vectors by Yingrun Biotechnologies, Inc. to generate the lentiviral vectors against HMGN5. The other shRNA sequences with no significant homology to any known human genes (5'-GACTTCATAAGGCGCATGC-3') were employed to generate the shRNA control lentiviral vectors. UBC cells were placed on 6 -well plates $\left(\sim 5 \times 10^{4}\right.$ cells/well $)$ until sufficient cell fusion, then the cells were infected with the recombinant lentivirus at a multiplicity of infection of 50, recommended by the manufacturer, for $24 \mathrm{~h}$. Medium was subsequently replaced with RPMI-1640 medium and $1 \mu \mathrm{g} / \mathrm{ml}$ puromycin (Sigma-Aldrich; Merck KGaA, Darmstadt, Germany) could be used for screening positively stable transfectants. Blank controls (untransfected controls) were used in experiments to demonstrate there was no significant difference between untransfected controls and cells transfected with shControl lentivirus.

CDDP was obtained from Sigma-Aldrich (Merck KGaA) and diluted in sterile serum (Thermo Fisher Scientific, Inc.) as indicated concentrations $(0,1,2,4,8$ and $16 \mu \mathrm{g} / \mathrm{ml})$. The PI3K signaling activator, insulin-like growth factor-1 (IGF-1), was purchased from PeproTech, Inc. (Rocky Hill, NJ, USA) and dissolved in dimethyl sulfoxide (DMSO) (15). Different concentrations $(0,1,2,4,8$ and $16 \mu \mathrm{g} / \mathrm{ml})$ of CDDP were used to treat different types of UBC cells or cells infected with lentivirus for dose-dependent cell proliferation assay for $72 \mathrm{~h}$. IGF-1 (50 ng/ml) was also added as indicated.
Western blot analysis. UBC cells were treated with CDDP $(6 \mu \mathrm{g} / \mathrm{ml})$, with or without IGF-1 $(50 \mathrm{ng} / \mathrm{ml})$, and then lysed with lysis buffer supplemented with protease inhibitor (catalog no. P0013B; Beyotime Institute of Biotechnology, Haimen, China). Western blotting was performed as previously described (10). In the present study, antibody against HMGN5 (dilution, 1:1,000; catalog no. ab18601) was obtained from Abcam (Cambridge, MA, USA), while antibodies against Akt (catalog no. 4685S), phosphorylated (p)-Akt (Ser ${ }^{473}$; catalog no. 4060P), slug (catalog no. 9585P), E-cadherin (catalog no. 3195S), VEGF-C (catalog no. 2445S), cytochrome c (catalog no. 19940S), cleaved-caspase-3 (catalog no. 9664P), cleaved-PARP (catalog no.5625P) and $\beta$-actin (catalog no. 4967; all 1:1,000) were purchased from Cell Signaling Technology, Inc. (Danvers, MA, USA). The proteins were visualized using the ECL Plus Kit (Thermo Fisher Scientific, Inc.). $\beta$-actin served as a loading control. Experiments were performed in triplicate.

Cell proliferation assay. Cell proliferation under CDDP treatment, with or without IGF-1, was analyzed by the MTT assay as previously described (10). Briefly, the cells were seeded on 96 -well plates $\left(4 \times 10^{3}\right.$ cells/well $)$ and treated with CDDP as aforementioned. After $24 \mathrm{~h}$, MTT $(5 \mathrm{mg} / \mathrm{ml}$; Sigma-Aldrich; Merck KGaA) was added to cells for $4 \mathrm{~h}$ at $37^{\circ} \mathrm{C}$. Subsequently, DMSO was used to dissolve the purple-blue formazan crystals produced by living cells. Absorbance was measured at $490 \mathrm{~nm}$.

Colony formation assay. After $72 \mathrm{~h}$ infection, 5637 cells were seeded at a low density of 400 cells/well onto a 6-well plate. RPMI-1640 medium was added with CDDP $(6 \mu \mathrm{g} / \mathrm{ml})$, with or without IGF-1 (50 ng/ml) as indicated, and changed every 3 days for 2 weeks. Colonies ( $\geq 50$ cells in a colony) were counted under a light microscope (magnification, x200; Leica TCS-SP5; Leica Microsystems GmbH, Wetzlar, Germany) subsequent to being fixed with pure methanol for $15 \mathrm{~min}$ at room temperature and stained with $0.005 \%$ gentian violet for $20 \mathrm{~min}$ at room temperature. Experiments were tested in triplicate.

Cell invasion assay. Cell invasion was evaluated using the Transwell assay as described previously (10). Briefly, the upper chamber of each Transwell insert (pore size, $8 \mu \mathrm{m}$; Corning Incorporated, Corning, NY, USA) was paved with the Matrigel gelatum (BD Biosciences, San Jose, CA, USA), in which approximately $1 \times 10^{5}$ bladder cancer 5637 cells with different HMGN5 expression were seeded. The cells were incubated at $37^{\circ} \mathrm{C}$ in serum-free RPMI-1640 medium medium and treated with CDDP $(6 \mu \mathrm{g} / \mathrm{ml})$ as well as IGF-1 (50 $\mathrm{ng} / \mathrm{ml}$ ). Subsequent to culturing for $24 \mathrm{~h}$ to allow cell migration, adherent cells in the upper chamber were removed with a cotton swab and the invasive cells attached on the lower surface were fixed with $4 \%$ paraformaldehyde for $30 \mathrm{~min}$ at room temperature. The cells were stained with $0.1 \%$ crystal violet for $20 \mathrm{~min}$ at room temperature and counted using a confocal microscope (magnification, x200, Leica TCS-SP5).

Cell apoptosis assay. Annexin V-fluorescein isothiocyanate apoptosis kit (Nanjing KeyGen Biotech Co. Ltd., Nanjing, 

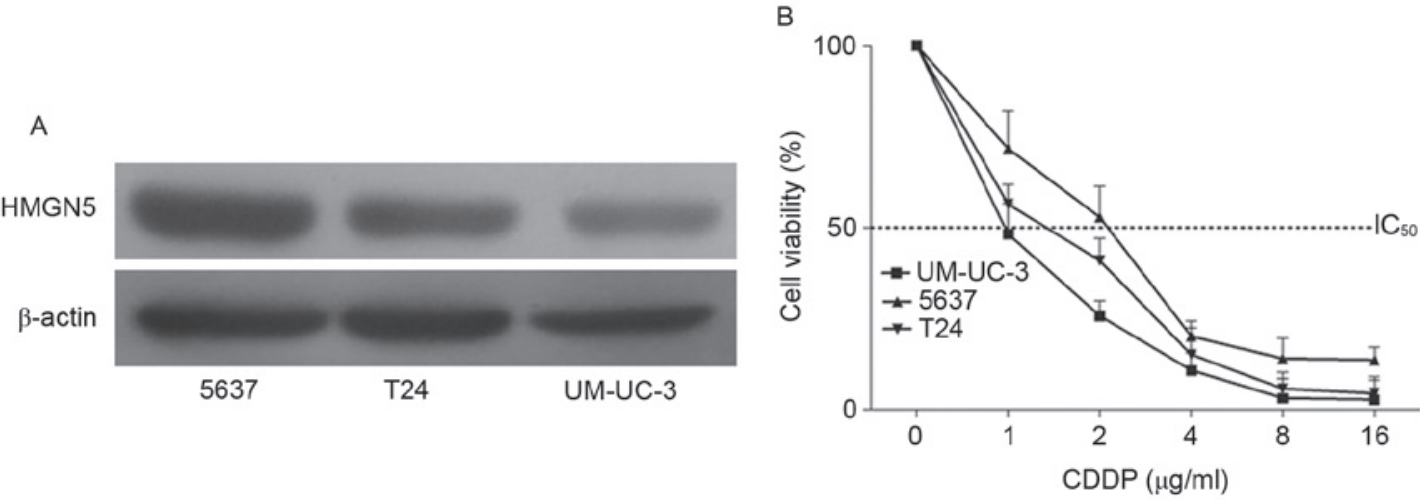

C

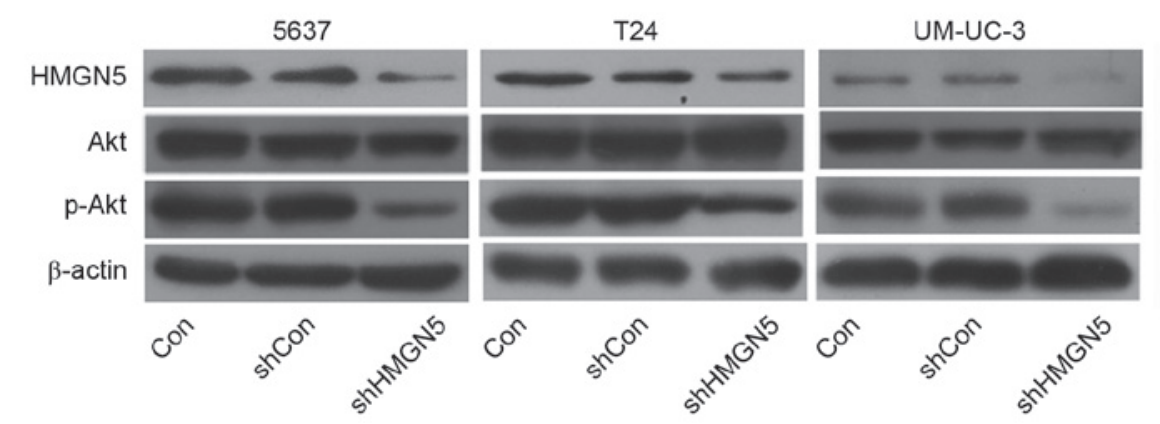

Figure 1. HMGN5 determines the responses of UBC cell lines to CDDP via the phosphoinositide 3-kinase/Akt signaling pathway. (A) Western blotting of HMGN5 expression in 5637, T24 and UM-UC-3 cell lines. $\beta$-actin served as a loading control. (B) Different concentrations of CDDP as indicated were used to treat 5637, T24 and UM-UC-3 cells for dose-dependent cell proliferation MTT assay for $72 \mathrm{~h}$. (C) 5637, T24 and UM-UC-3 cells were infected with lentivirus for HMGN5 depletion or negative control, the expression of HMGN5, Akt and p-Akt was subsequently analyzed by western blotting after $72 \mathrm{~h}$. $\beta$-actin served as a loading control. Results are expressed as the mean \pm standard deviation $(n=3)$. HMGN5, high mobility group nucleosome-binding domain 5; CDDP, cisplatin; p-, phosphorylated; $\mathrm{IC}_{50}$, Half-maximal inhibitory concentration; sh, short hairpin; Con, control.

China) was used to measure apoptosis as reported previously (10). Briefly, following infection and drug treatment with CDDP $(6 \mu \mathrm{g} / \mathrm{ml})$ and IGF-1 $(50 \mathrm{ng} / \mathrm{ml})$ for $24 \mathrm{~h}$, the cells were collected and labeled with annexin $\mathrm{V}$ as well as propidium iodide (PI) in the dark. Cell apoptosis was then detected using a FACSCalibur flow cytometry (BD Biosciences, San Jose, CA, USA). The results were analyzed using BD FACSDiva 6.1.3 software (BD Biosciences).

Hoechst 33342 dye. Following transfection, 5637 cells were cultured on a 24-well plate and treated with CDDP and IGF-1 as aforementioned. Hoechst $33342(1 \mu \mathrm{g} / \mathrm{ml}$; Thermo Fisher Scientific, Inc.) was then added, and the cells were stained for $20-30 \mathrm{~min}$ at $37^{\circ} \mathrm{C}$. Subsequently, the cells were washed twice with ice-cold PBS and observed under a fluorescence microscope (magnification, x200, Leica TCS-SP5). Thick and dense fluorescence was observed in the apoptotic cells. The percentage of apoptotic cells was calculated according to the following formula: (Apoptotic cells in five random visual fields)/(total cells in five random visual fields).

Statistical analysis. All data were collected from three independent experiments and analyzed using GraphPad Prism 5.01 software (GraphPad Software, Inc., La Jolla, CA, USA). The quantitative data are presented as the mean \pm standard deviation. Differences among the means of multiple groups were compared by one-way analysis of variance, followed by a Newman-Keuls multiple comparison test. $\mathrm{P}<0.05$ was considered to indicate a statistically significant difference.

\section{Results}

HMGN5 protein determines the response of UBC cells to $C D D P$. The expression of HMGN5 protein was determined using western blotting in UBC cell lines 5637, T24 and UM-UC-3 cells. The results confirmed that the level of HMGN5 protein in 5637 cells was the highest among the three cell lines, while the level in UM-UC-3 cells was the lowest (Fig. 1A). Cell proliferation MTT assays were then employed to investigate the chemosensitivity of the three cell lines to CDDP. Following incubation with the indicated concentrations of CDDP for $72 \mathrm{~h}$, it was revealed that the 5637 cells were relatively more resistant to CDPP compared with the other two types of cells (Fig. 1B). Notably, the UM-UC-3 cells exhibited the highest sensitivity in the three cell types (Fig. 1B).

The PI3K/Akt signaling pathway is associated with the development of resistance to CDDP in human malignancies (16). Zhou et al (17) first reported that HMGN5 is involved in the progression of osteosarcoma through PI3K/Akt signaling. The present study also confirmed that, following successful knockdown of HMGN5 in UBC cell lines, the expression of p-Akt was subsequently inhibited (Fig. 1C). These results indicated that HMGN5 might be involved in the negative regulation of chemosensitivity in UBC cell lines to CDDP via the PI3K/Akt signaling pathway.

HMGN5 is a molecular target of CDDP treatment in 5637 cells. To further investigate the molecular mechanisms 


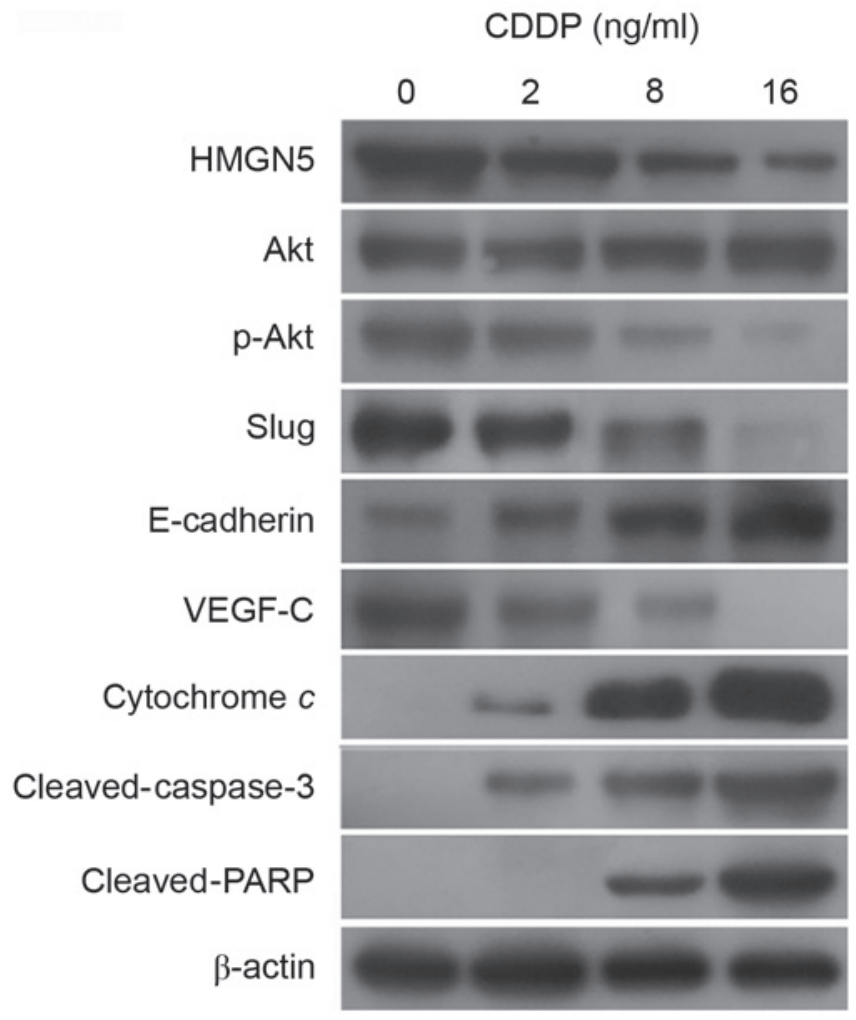

Figure 2. HMGN5 serves as a target for CDDP treatment in UBC cells. Different concentrations $(0,1,2,8$ and $16 \mu \mathrm{g} / \mathrm{ml})$ of CDDP were used to treat UBC 5637 cells for $24 \mathrm{~h}$. The expression of HMGN5 and its downstream signal molecules, including Akt, p-Akt, slug, E-cadherin and VEGF-C, was then analyzed by western blot analysis. The expression of a number of markers in the mitochondrial apoptosis pathway, including cytochrome $\mathrm{c}$, cleaved-caspase- 3 and cleaved-PARP, was also analyzed. $\beta$-actin served as a loading control. HMGN5, high mobility group nucleosome-binding domain 5; CDDP, cisplatin; p-, phosphorylated; PARP, poly[ADP-ribose] synthase 1; VEGF, vascular endothelial growth factor.

underlying the involvement of HMGN5 in CDDP treatment, the expression of HMGN5, Akt, p-Akt, VEGF-C, slug and E-cadherin was analyzed in 5637 cells during CDDP treatment for $24 \mathrm{~h}$. Additionally, considering the participation of mitochondrial apoptosis pathway in cellular response in tumor cells to numerous anticancer drugs, including CDDP (18), the expression of cytochrome $\mathrm{c}$, cleaved-caspase- 3 and cleaved-PARP was also detected.

The results from western blot analysis indicated that CDDP impaired the expression of HMGN5 protein in a dose-dependent manner (Fig. 2). Furthermore, consistent with the change in HMGN5 protein expression, the active form of Akt, p-Akt, was also downregulated by CDDP treatment (Fig. 2). According to previous studies, the expression of VEGF-C, slug and the process of EMT can be positively regulated by $\mathrm{PI} 3 \mathrm{~K} / \mathrm{Akt}$ signaling (19-21). Consistent with these findings, in the present study, the expression of VEGF-C and slug was observed to be inhibited by CDDP treatment, while the expression of the epithelial marker, E-cadherin, was increased (Fig. 2). Furthermore, the expression of cytochrome c, cleaved-caspase-3 and cleaved-PARP was increased in a dose-dependent manner during CDDP treatment, indicating the activation of the mitochondrial apoptosis pathway by CDDP treatment (Fig. 2).
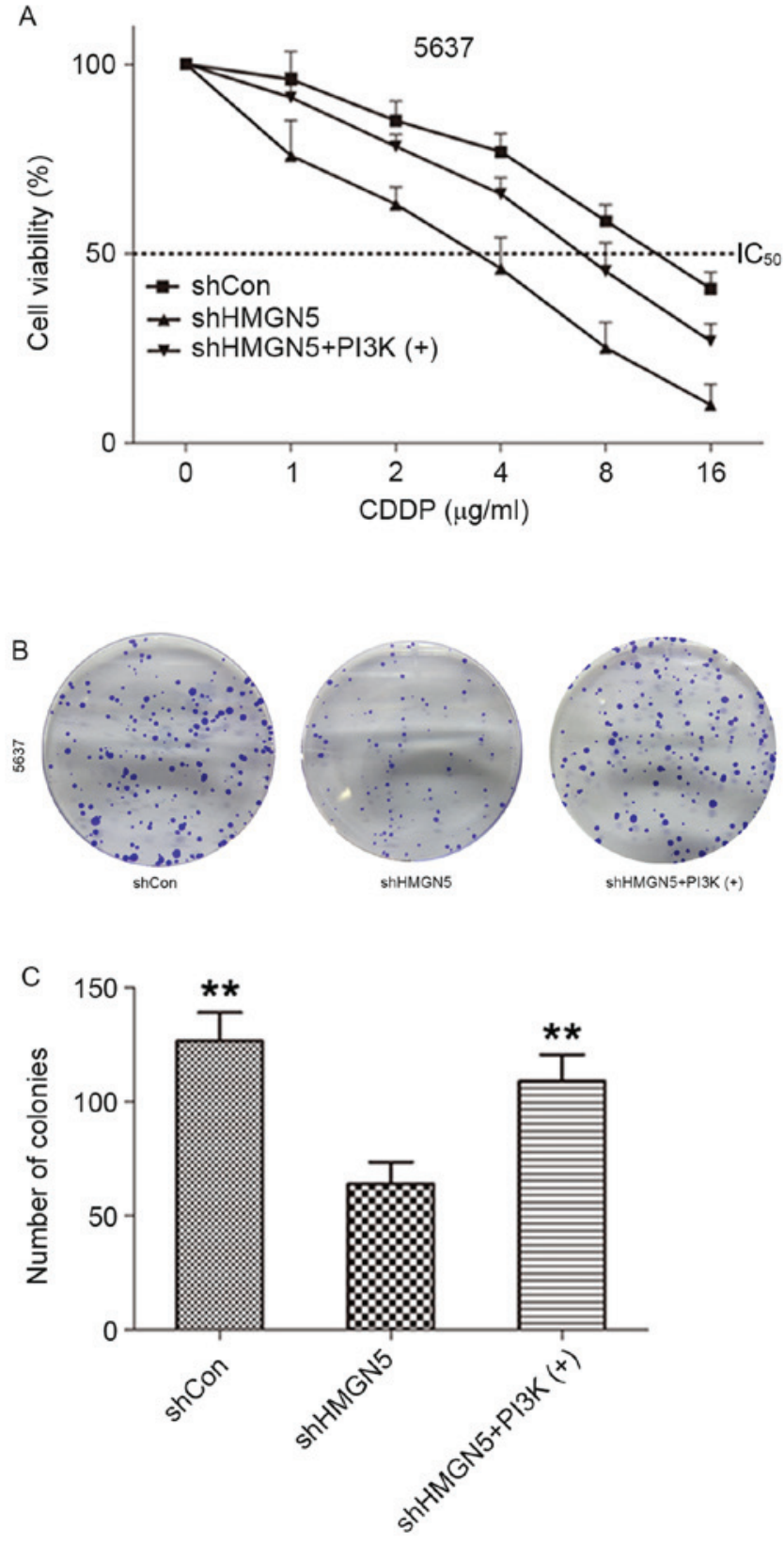

Figure 3. HMGN5 depletion impairs the viability and clonogenicity in UBC cells. (A) Dose-dependent MTT assay was employed to investigate the effect of HMGN5-knockdown on viability of 5637 cells and the effect of IGF-1 (a PI3K signaling activator) on the viability of 5637 cells with HMGN5-knockdown following CDDP treatment for $24 \mathrm{~h}$. (B) Colony formation assay was utilized to detect the effect of HMGN5-knockdown on the clonogenicity in 5637 cells and the effect of IGF-1 on the clonogenicity in 5637 cells with HMGN5 inhibition. (C) Quantitative summary of colony formation in different groups. Results are expressed as the mean \pm standard deviation $(\mathrm{n}=3),{ }^{* * *} \mathrm{P}<0.01$ compared with HMGN5-knocked down 5637 cells. CDDP, cisplatin; HMGN5, high mobility group nucleosome-binding domain 5; IGF-1, insulin-likegrowth factor-1; PI3K, phosphoinositide 3-kinase; sh, short hairpin; Con, control; $\mathrm{IC}_{50}$, Half-maximal inhibitory concentration.

Knockdown of HMGN5 increases the chemosensitivity of 5637 cells to CDDP. To verify the function of HMGN5 in controlling sensitivity of UBC cells to CDDP and elucidate the mechanisms, the 5637 cell line was used as the cell model and the loss-of-function method was employed. As demonstrated in Fig. 3A, following incubation with various concentrations 


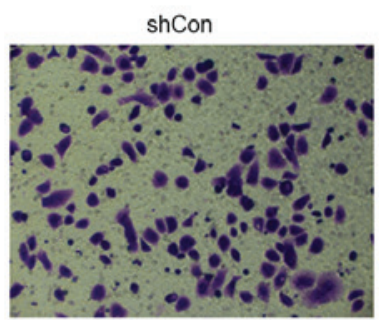

ShHMGN5

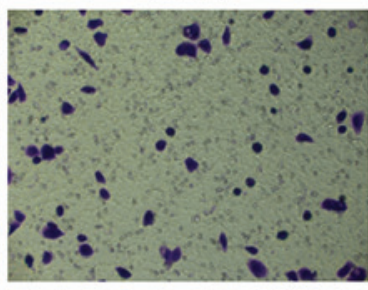

shHMGN5+PI3K (+)

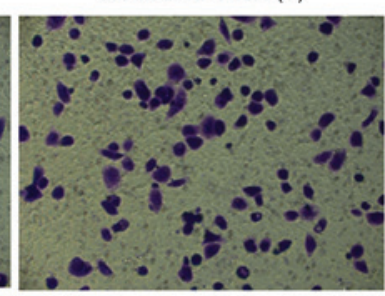

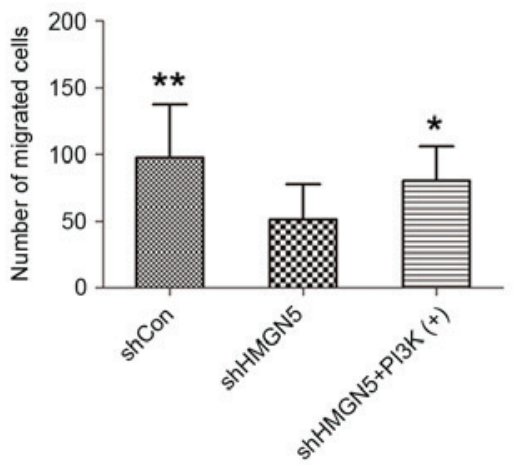

B

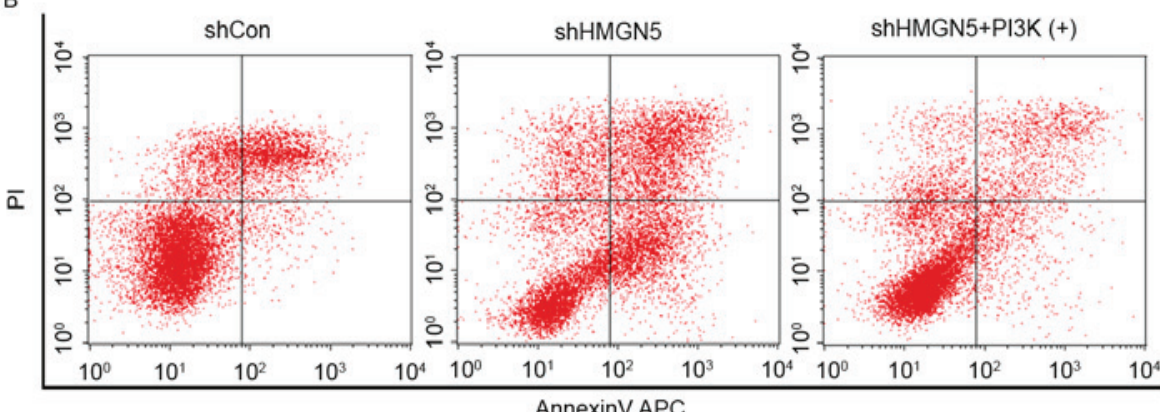

AnnexinV APC

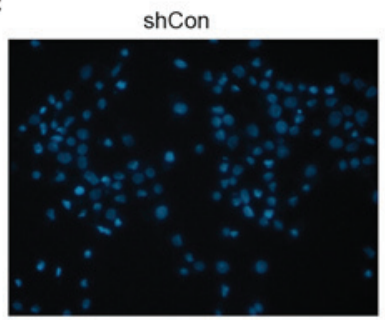

shHMGN5

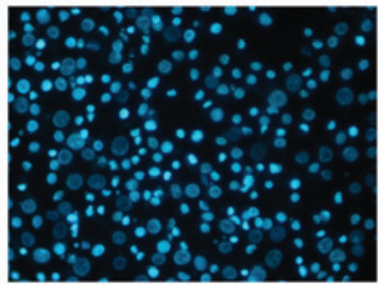

shHMGN5+PI3K (+)

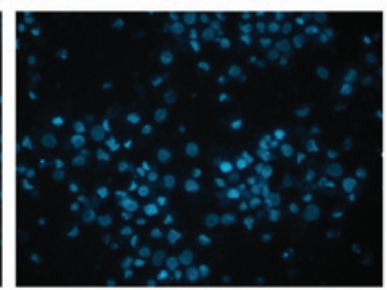

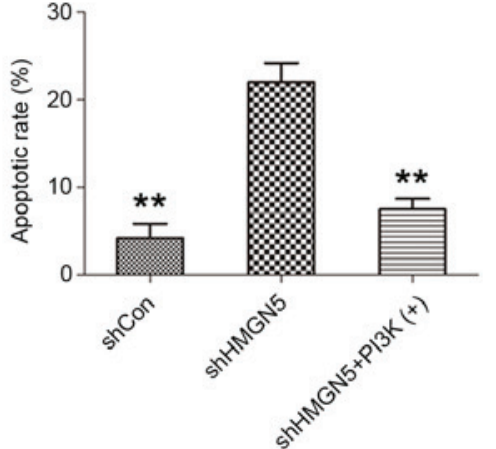

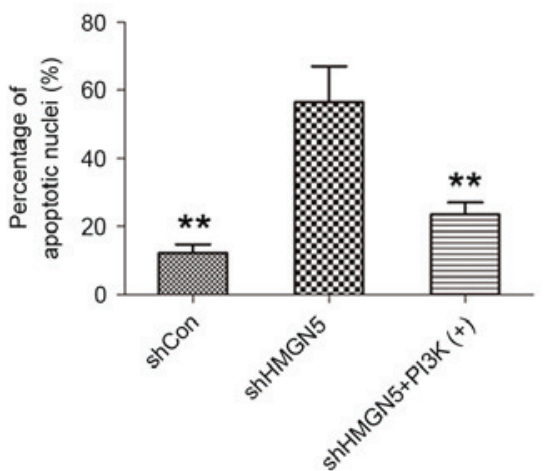

Figure 4. HMGN5-knockdown inhibits invasion but increases apoptosis in UBC cells. (A) Following infection with lentivirus, 5637 cells were treated with CDDP, with or without IGF-1 (left panels). The Transwell assay was then used to detect cell invasion. The cells penetrating the Matrigel were stained with crystal violet and shown as blue under a light microscope (magnification, x200). Quantitative analysis of the number of migrated cells in different groups (right panel). (B) After infection, the cells were treated with indicated agents for $24 \mathrm{~h}$, followed by staining with Annexin V-fluorescein isothiocyanate and PI and analyzed by flow cytometry (left panels). Quantitative analysis of the rate of early apoptosis in different groups (right panel). (C) Hoechst 33342 staining was employed to observe the morphological change of apoptotic cells (left panels; magnification, x200). Cell nuclei with apoptosis were characterized by pyknosis, shrink and karyorrhexis and exhibited the marked fluorescence under fluorescence microscope. Quantitative analysis of the percentage of apoptotic nuclei in different groups (right panel). Results are expressed as the mean \pm standard deviation $(\mathrm{n}=3)$. ${ }^{*} \mathrm{P}<0.05,{ }^{* *} \mathrm{P}<0.01$ compared with $\mathrm{HMGN5}$ knocked down 5637 cells. HMGN5, high mobility group nucleosome-binding domain 5; PI3K, phosphoinositide 3-kinase; sh, short hairpin; Con, control; PI, propidium iodide.

(1-16 $\mu \mathrm{g} / \mathrm{ml})$ of CDDP for $24 \mathrm{~h}$, knockdown of HMGN5 markedly decreased viability of 5637 cells compared with negative control cells. As expected, the effect could be reversed to a certain extent by treatment with the PI3K signaling activator, IGF-1. Similarly, the results from colony formation assay indicated that 5637 cells where HMGN5 was knocked down formed fewer and smaller colonies compared with the negative control cells in monolayer culture for 14 days, which could also be rescued to a certain extent by IGF-1 treatment (Fig. 3B and $\mathrm{C}$ ). These results indicated that HMGN5-knockdown might increase the therapeutic effects of CDDP in inhibiting the proliferation and tumorigenesis of 5637 cells.

Transwell invasion assays were subsequently employed to investigate the effects of HMGN5-knockdown on invasion of UBC cells following CDDP treatment for $24 \mathrm{~h}$.
HMGN5-knockdown was found to significantly decrease the number of 5637 cells crossing the Matrigel, indicating that HMGN5-knockdown inhibits the invasiveness of UBC cells during CDDP treatment (Fig. 4A). In addition, this effect on invasion can be blocked by IGF-1 to a certain extent (Fig. 4A). Flow cytometry and cell nuclei Hoechst 33342 staining were also utilized to detect cell apoptosis following CDDP treatment for $24 \mathrm{~h}$. The results from flow cytometry demonstrated that the early apoptotic rate was increased by HMGN5-knockdown in 5637 cells, and this may be reversed by treatment IGF-1 to a certain extent (Fig. 4B). Furthermore, as shown in Fig. 4C, morphological changes in the nuclei of apoptotic cells were analyzed using Hoechst 33342 staining, which are characterized by pyknosis, shrinkage and karyorrhexis, with marked fluorescence (Fig. 4C). It was revealed that the percentage 


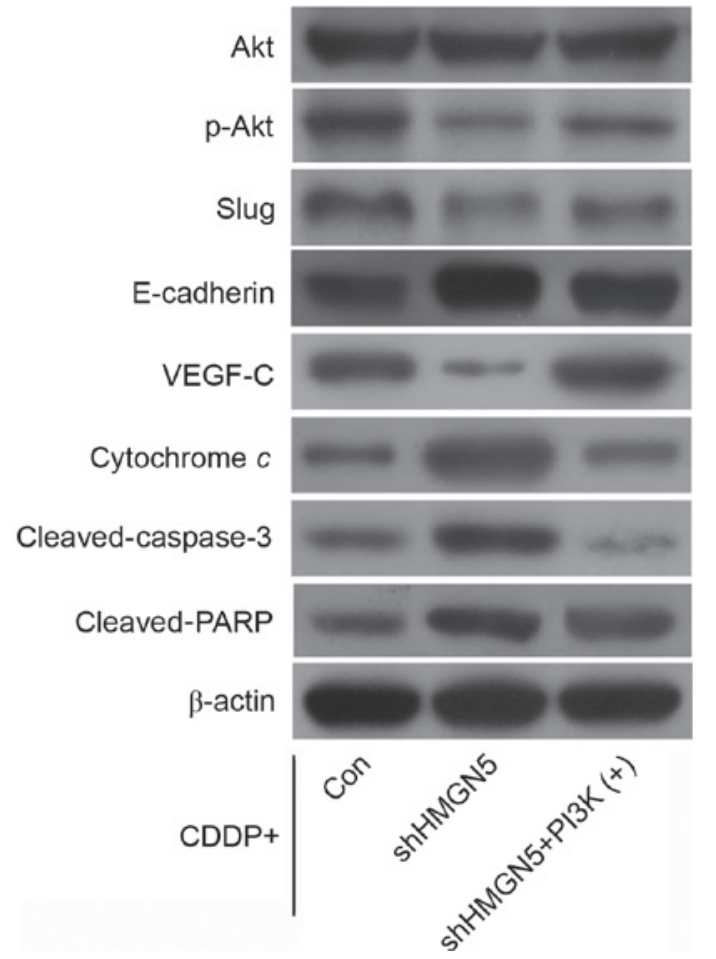

Figure 5. Effect of HMGN5 depletion on the expression of Akt, p-Akt, slug, E-cadherin, VEGF-C, cytochrome c, cleaved-caspase-3 and cleaved-PARP. After infection with lentivirus as indicated, 5637 cells were treated with CDDP, with or without IGF-1. The expression of Akt, p-Akt, slug, E-cadherin, VEGF-C, cytochrome c, cleaved-caspase- 3 and cleaved-PARP was then analyzed by western blotting. $\beta$-actin served as a loading control. CDDP, cisplatin; IGF-1, insulin-like growth factor-1; PARP, poly ADP ribose polymerase; VEGF, vascular endothelilal growth factor; HMGN5, high mobility group nucleosome-binding domain 5 .

of apoptotic nuclei in the HMGN5 knocked down group was significantly higher compared with the percentage in the negative control group and the IGF-1 treatment group (Fig. 4C).

Additionally, western blotting indicated that HMGN5-knockdown decreased the expression of p-Akt and VEGF-C compared with control, and increased the expression of E-cadherin, cytochrome c, cleaved-caspase-3 and cleaved-PARP during CDDP treatment. Notably, it was possible to reverse these changes in protein expression by IGF-1 treatment (Fig. 5).

\section{Discussion}

HMGN5 is ubiquitously expressed in human tissues and is classified as a member of the HMGN family of proteins, based on the presence of the three important functional domains: The nucleosome-binding domain, the nuclear localization signal and the negatively charged C-terminus (8). On account of these domains, HMGN5 is able to enter the nucleus, bind to nucleosomes, unfold chromatin and increase the accessibility of DNA (8). As a result, factors associated with DNA lesion and repair, replication, transcription and recombination may combine with DNA more easily and take action and thereby modulate the cellular epigenetic profile (for example, ultraviolet light can cause more DNA lesions when chromatin is unfolded) $(8,22,23)$. In the last few years, ectopic high expression level of HMGN5 has been confirmed in several malignant tumors, including prostate cancer, renal cancer, lung cancer, breast cancer, osteosarcoma and glioma, indicating the potential role of this protein as a target in cancer therapy $(9,11)$. The present authors previously identified the carcinogenic role of HMGN5 in UBC and reported the regulation of the gene in the expression of E-cadherin and VEGF-C $(10,11,24)$. In the present study, the mechanisms underlying the modulation were further investigated and the possible application of HMGN5 in UBC therapy was examined.

Zhou et al (17) highlighted that, in osteosarcoma U2-OS and $\mathrm{SaO} 2$ cell lines, HMGN5 positively regulates the expression of PI3Kp85 $\alpha$ and p-Akt and contributes to the malignant potential of tumor cells. Similar to the results from the study by Zhou et al (17), in the present study it was observed that the expression of p-Akt was subsequently decreased with no change in Akt expression following the silencing of HMGN5 in UBC 5637, T24 and UM-UC-3. These findings indicate that HMGN5 may positively regulate PI3K/Akt signaling. The possible mechanism may be that HMGN5 can act as a protein kinase and lead to phosphorylation of Akt (25). However, the mechanism can also be indirect as HMGN5 may amplify the transcriptional level of the catalytic subunit in PI3K, therefore, additional studies are required to completely explain this effect (23). Furthermore, as reported, the progression of EMT and the expression of VEGF-C can be regulated by PI3K/Akt signaling, therefore, it was hypothesized that HMGN5 may modulate E-cadherin and VEGF-C expression through PI3K/Akt signaling (19-21).

Chemotherapy represents an important approach in the comprehensive treatment of solid tumors, while the efficacy usually depends on the sensitivity of tumor cells to chemotherapeutics. In fact, numerous studies support the pivotal role of certain oncogenes in the regulation of chemosensitivity, such as transcription factor AP- $2 \alpha$ in UBC cells and REV3-like DNA-directed polymerase $\zeta$ catalytic subunit in cervical cancer cells $(26,27)$. The role of HMGN5 in tumorigenesis in a number of types of cancer has been highlighted (9). However, information regarding HMGN5 in the regulation of chemosensitivity is insufficient and disputable. He et al (28) reported that the inhibition of HMGN5 sensitized the malignant meningioma IOMM-Lee and CH157 cells to temozolomide-induced cytotoxicity through modulation of the expression of multidrug resistance-associated protein-1, B cell lymphoma-2 and cleaved-caspase-3. Similarly, in a study on osteosarcoma cells, Zhou et al (17) observed that the knockdown of HMGN5 enabled tumor cells to be significantly more sensitive to doxorubicin-induced cell injury via activating the apoptotic signaling pathways. These findings indicated that HMGN5 knockdown may contribute to the increase in chemosensitivity to antitumor drugs. Guo et al (29) revealed that HMGN5 expression was positively associated with chemosensitivity of prostate cancer cell lines to gemcitabine. In addition, they also demonstrated that HMGN5 depletion reduced the sensitivity of PC-3 cells to gemcitabine, while HMGN5 overexpression in DU145 cells increased the sensitivity to the agent (29). Therefore, a high expression level of HMGN5 may be beneficial to patients with chemotherapy.

As one of the most effective anticancer drugs, CDDP has been extensively used for the treatment of different types of cancer, including UBC (18). The present data supported the 
view from Zhou et al (17) and He et al (28), as it was revealed from MTT assays that UBC cells with lower levels of HMGN5 expression were relatively more sensitive to CDDP compared with cells with higher levels of expression. Furthermore, the inhibition of HMGN5 was able to markedly decrease proliferation, colony formation and invasion of 5637 cells during CDDP treatment, indicating that depletion of the HMGN gene in UBC cells with relatively high HMGN5 expression may increase the sensitivity of tumor cells to CDDP.

Abnormal activation of the PI3K/Akt signaling pathway has been reported to have critical roles in the development of resistance to CDDP, while inhibition of PI3K/Akt signaling increases the effect of CDPP $(30,31)$. In addition, the progression of EMT and overexpression of its activators, such as slug, are positively associated with CDDP resistance $(12,13)$. VEGF-C, a marker of lymphangiogenesis, is also involved in the regulation of CDDP sensitivity (14). In the present study, western blotting results indicated that the level of p-Akt, slug, E-cadherin and VEGF-C proteins was inhibited in 5637 cells where HMGN5 was knocked down and treated with CDDP, indicating the involvement of PI3K/Akt signaling, EMT and lymphangiogenesis. As reported, activation of cell apoptosis also serves as a major mechanism underlying the anticancer effects of CDDP $(18,32)$. In the present study, the results from flow cytometric analysis and Hoechst 33342 staining demonstrated that cell apoptosis was enhanced in 5637 cells where HMGN5 was depleted compared to cells transfected with the shRNA control vector.

Cell apoptosis is executed by members of the caspase family, which can be activated by the mitochondrial apoptotic pathway (33). Inactivation of PI3K/Akt signaling leads to the release of cytochrome $\mathrm{c}$ from the mitochondria to the cytoplasm, which leads to the activation of caspase-3 and PARP, and subsequently triggers apoptosis (34). In the present study, increased expression of cytochrome c, cleaved-caspase-3 and cleaved-PARP was observed in 5637 cells where HMGN5 was knocked down and treated with CDDP, which confirms the activation of the mitochondrial apoptotic pathway. The changes caused by the inhibition of HMGN5 in 5637 cells were able to be reversed by treatment with the PI3K/Akt signaling activator IGF-1, indicating a mechanism in which HMGN5 positively regulates the activity of the PI3K/Akt signaling pathway.

Notably, CDDP was previously observed to impair the expression of HMGN5 and therefore able to affect its downstream signal molecules. A potential explanation may be that HMGN5 can serve as a molecular target for CDDP, and therefore CDDP is able to directly inhibit HMGN5 in UBC cells (29). Another explanation may be that CDDP is able to kill the UBC cells, which then leads to a decrease in HMGN5 expression. However, the mechanism remains unclear and required to be further investigated.

Although certain limitations were inevitable, including the lack of in vivo experiments and the experimental data from HMGN5-overexpressing bladder cancer cells, a number of primary findings were reported. Based on results of the present study, it was concluded that HMGN5 regulates the PI3K/Akt signaling pathway in UBC cell lines and determines the response of UBC cell lines to CDDP. Through inhibiting PI3K/Akt signaling, knock down of HMGN5 increases the chemosensitivity of UBC cells to CDDP. Therefore, HMGN5 may be a promising therapeutic target for the treatment of UBC and hinder its progression.

\section{Acknowledgements}

The present study was supported by the Hunan Provincial Natural Science Foundation of China (grant no. 14JJ3044) and the China Scholarship Council (grant no. 201606370204).

\section{References}

1. Siegel RL, Miller KD and Jemal A: Cancer statistics, 2016. CA Cancer J Clin 66: 7-30, 2016.

2. Burger M, Catto JW, Dalbagni G, Grossman HB, Herr H, Karakiewicz P, Kassouf W, Kiemeney LA, La Vecchia C, Shariat S, et al: Epidemiology and risk factors of urothelial bladder cancer. Eur Urol 63: 234-241, 2013.

3. Kaufman DS, Shipley WU and Feldman AS: Bladder cancer. Lancet 374: 239-249, 2009.

4. Jacobs BL, Lee CT and Montie JE: Bladder cancer in 2010: How far have we come? CA Cancer J Clin 60: 244-272, 2010.

5. von der Maase H, Sengelov L, Roberts JT, Ricci S, Dogliotti L, Oliver T, Moore MJ, Zimmermann A and Arning M: Long-term survival results of a randomized trial comparing gemcitabine plus cisplatin, with methotrexate, vinblastine, doxorubicin, plus cisplatin in patients with bladder cancer. J Clin Oncol 23: 4602-4608, 2005 .

6. Vaishampayan U: Systemic therapy of advanced urothelial cancer. Curr Treat Options Oncol 10: 256-266, 2009.

7. King LM and Francomano CA: Characterization of a human gene encoding nucleosomal binding protein NSBP1. Genomics 71: 163-173, 2001.

8. Rochman M, Malicet C and Bustin M: HMGN5/NSBP1: A new member of the HMGN protein family that affects chromatin structure and function. Biochim Biophys Acta 1799: 86-92, 2010.

9. Shi Z, Tang R, Wu D and Sun X: Research advances in HMGN5 and cancer. Tumour Biol 37: 1531-1539, 2016.

10. Gan Y, Tan J, Yang J, Zhou Y, Dai Y, He L, Yao K and Tang Y: Knockdown of HMGN5 suppresses the viability and invasion of human urothelial bladder cancer 5637 cells in vitro and in vivo. Med Oncol 32: 136, 2015

11. Yao K, He L, Gan Y,Zeng Q, Dai Y and Tan J: MiR-186 suppresses the growth and metastasis of bladder cancer by targeting NSBP1. Diagn Pathol 10: 146, 2015

12. Haslehurst AM, Koti M, Dharsee M, Nuin P, Evans K, Geraci J, Childs T, Chen J, Li J, Weberpals J, et al: EMT transcription factors snail and slug directly contribute to cisplatin resistance in ovarian cancer. BMC Cancer 12: 91, 2012.

13. Zhao J, Dong D, Sun L, Zhang G and Sun L: Prognostic significance of the epithelial-to-mesenchymal transition markers e-cadherin, vimentin and twist in bladder cancer. Int Braz J Urol 40: 179-189, 2014.

14. Zhu H, Yun F, Shi X and Wang D: VEGF-C inhibition reverses resistance of bladder cancer cells to cisplatin via upregulating maspin. Mol Med Rep 12: 3163-3169, 2015.

15. Gan Y, Wang Y, Tan Z, Zhou J, Kitazawa R, Jiang X, Tang Y and Yang J: TDRG1 regulates chemosensitivity of seminoma TCam-2 cells to cisplatin via PI3K/Akt/mTOR signaling pathway and mitochondria-mediated apoptotic pathway. Cancer Biol Ther 17: 741-750, 2016.

16. West KA, Castillo SS and Dennis PA: Activation of the PI3K/Akt pathway and chemotherapeutic resistance. Drug Resist Updat 5: 234-248, 2002.

17. Zhou X, Yuan B, Yuan W, Wang C, Gao R and Wang J: The expression and clinical significance of high mobility group nucleosome binding domain 5 in human osteosarcoma. Tumour Biol 35: 6539-6547, 2014.

18. Dasari S and Tchounwou PB: Cisplatin in cancer therapy: Molecular mechanisms of action. Eur J Pharmacol 740: 364-378, 2014

19. Liang W, Hao Z, Han JL, Zhu DJ, Jin ZF and Xie WL: CAV-1 contributes to bladder cancer progression by inducing epithelial-to-mesenchymal transition. Urol Oncol 32: 855-863, 2014.

20. Wissmann C and Detmar M: Pathways targeting tumor lymphangiogenesis. Clin Cancer Res 12: 6865-6868, 2006. 
21. Chen H, Guan R, Lei Y, Chen J, Ge Q, Zhang X, Dou R, Chen H, Liu H, Qi X, et al: Lymphangiogenesis in gastric cancer regulated through Akt/mTOR-VEGF-C/VEGF-D axis. BMC Cancer 15: $103,2015$.

22. Gerlitz G: HMGNs, DNA repair and cancer. Biochim Biophys Acta 1799: 80-85, 2010

23. Rochman M, Postnikov Y, Correll S, Malicet C, Wincovitch S, Karpova TS, McNally JG, Wu X, Bubunenko NA, Grigoryev S, et al: The interaction of NSBP1/HMGN5 with nucleosomes in euchromatin counteracts linker histone-mediated chromatin compaction and modulates transcription. Mol Cell 35: 642-656, 2009.

24. Wahafu W, He ZS, Zhang XY, Zhang CJ, Yao K, Hao H, Song G, He Q, Li XS and Zhou LQ: The nucleosome binding protein NSBP1 is highly expressed in human bladder cancer and promotes the proliferation and invasion of bladder cancer cells. Tumour Biol 32: 931-939, 2011.

25. Burgering BM and Coffer PJ: Protein kinase B (c-Akt) in phosphatidylinositol-3-OH kinase signal transduction. Nature 376 : 599-602, 1995.

26. Nordentoft I, Dyrskjot L, Bodker JS, Wild PJ, Hartmann A, Bertz S, Lehmann J, Orntoft TF and Birkenkamp-Demtroder K: Increased expression of transcription factor TFAP2alpha correlates with chemosensitivity in advanced bladder cancer. BMC Cancer 11: 135, 2011.

27. Yang L, Shi T, Liu F, Ren C, Wang Z, Li Y, Tu X, Yang G and Cheng X: REV3L, a promising target in regulating the chemosensitivity of cervical cancer cells. PloS One 10: e0120334, 2015.
28. He J, Liu C, Wang B, Li N, Zuo G and Gao D: HMGN5 blockade by siRNA enhances apoptosis, suppresses invasion and increases chemosensitivity to temozolomide in meningiomas. Int J Oncol 47: 1503-1511, 2015.

29. Guo Z, Zhang X, Li X, Xie F, Su B, Zhang M and Zhou L: Expression of oncogenic HMGN5 increases the sensitivity of prostate cancer cells to gemcitabine. Oncol Rep 33: 1519-1525, 2015.

30. Zhang HY, Zhang PN and Sun H: Aberration of the $\mathrm{PI} 3 \mathrm{~K} / \mathrm{AKT} / \mathrm{mTOR}$ signaling in epithelial ovarian cancer and its implication in cisplatin-based chemotherapy. Eur J Obstet Gynecol Reprod Biol 146: 81-86, 2009.

31. Sun D, Sawada A, Nakashima M, Kobayashi T, Ogawa O and Matsui Y: MK2206 potentiates cisplatin-induced cytotoxicity and apoptosis through an interaction of inactivated Akt signaling pathway. Urol Oncol 33: 111.e17-111.e26, 2015.

32. Chang F, Lee JT, Navolanic PM, Steelman LS, Shelton JG, Blalock WL, Franklin RA and McCubrey JA: Involvement of PI3K/Akt pathway in cell cycle progression, apoptosis, and neoplastic transformation: A target for cancer chemotherapy. Leukemia 17: 590-603, 2003.

33. Nicholson DW and Thornberry NA: Apoptosis. Life and death decisions. Science 299: 214-215, 2003.

34. Estaquier J, Vallette F, Vayssiere JL and Mignotte B: The mitochondrial pathways of apoptosis. Adv Exp Med Biol 942: 157-183, 2012. 